$\mathrm{J}$ o u r n a l of

Mathematics

and Applications

JMA No 38, pp 33-48 (2015)

\title{
On some differential sandwich theorems using an extended generalized Sălăgean operator and extended Ruscheweyh operator
}

\author{
Loriana Andrei
}

\begin{abstract}
In this work we define a new operator using the extended generalized Sălăgean operator and extended Ruscheweyh operator. Denote by $D R_{\lambda}^{m, n}$ the Hadamard product of the extended generalized Sălăgean operator $D_{\lambda}^{m}$ and extended Ruscheweyh operator $R^{n}$, given by $D R_{\lambda}^{m, n}: \mathcal{A}_{\zeta}^{*} \rightarrow \mathcal{A}_{\zeta}^{*}, D R_{\lambda}^{m, n} f(z, \zeta)=\left(D_{\lambda}^{m} * R^{n}\right) f(z, \zeta)$ and $\mathcal{A}_{n \zeta}^{*}=\left\{f \in \mathcal{H}(U \times \bar{U}), f(z, \zeta)=z+a_{n+1}(\zeta) z^{n+1}+\ldots, z \in U, \zeta \in \bar{U}\right\}$ is the class of normalized analytic functions with $\mathcal{A}_{1 \zeta}^{*}=\mathcal{A}_{\zeta}^{*}$. The purpose of this paper is to introduce sufficient conditions for strong differential subordination and strong differential superordination involving the operator $D R_{\lambda}^{m, n}$ and also to obtain sandwich-type results.
\end{abstract}

AMS Subject Classification: 30C45

Keywords and Phrases: analytic functions, differential operator, differential subordination, differential superordination.

\section{Introduction}

Denote by $U$ the unit disc of the complex plane $U=\{z \in \mathbb{C}:|z|<1\}, \bar{U}=\{z \in$ $\mathbb{C}:|z| \leq 1\}$ the closed unit disc of the complex plane and $\mathcal{H}(U \times \bar{U})$ the class of analytic functions in $U \times \bar{U}$.

Let

$$
\mathcal{A}_{n \zeta}^{*}=\left\{f \in \mathcal{H}(U \times \bar{U}), f(z, \zeta)=z+a_{n+1}(\zeta) z^{n+1}+\ldots, z \in U, \zeta \in \bar{U}\right\},
$$

with $\mathcal{A}_{1 \zeta}^{*}=\mathcal{A}_{\zeta}^{*}$, where $a_{k}(\zeta)$ are holomorphic functions in $\bar{U}$ for $k \geq 2$, and $\mathcal{H}^{*}[a, n, \zeta]=\left\{f \in \mathcal{H}(U \times \bar{U}), f(z, \zeta)=a+a_{n}(\zeta) z^{n}+a_{n+1}(\zeta) z^{n+1}+\ldots, z \in U\right.$, $\zeta \in \bar{U}$, for $a \in \mathbb{C}$ and $n \in \mathbb{N}, a_{k}(\zeta)$ are holomorphic functions in $\bar{U}$ for $k \geq n$.

COPYRIGHT (c) by Publishing Department Rzeszów University of Technology P.O. Box 85, 35-959 Rzeszów, Poland 
Generalizing the notion of differential subordinations, J.A. Antonino and S. Romaguera have introduced in [17] the notion of strong differential subordinations, which was developed by G.I. Oros and Gh. Oros in [18].

Definition 1.1 [18] Let $f(z, \zeta), H(z, \zeta)$ analytic in $U \times \bar{U}$. The function $f(z, \zeta)$ is said to be strongly subordinate to $H(z, \zeta)$ if there exists a function $w$ analytic in $U$, with $w(0)=0$ and $|w(z)|<1$ such that $f(z, \zeta)=H(w(z), \zeta)$ for all $\zeta \in \bar{U}$. In such a case we write $f(z, \zeta) \prec \prec H(z, \zeta), z \in U, \zeta \in \bar{U}$.

Remark $1.1[18]$ (i) Since $f(z, \zeta)$ is analytic in $U \times \bar{U}$, for all $\zeta \in \bar{U}$, and univalent in $U$, for all $\zeta \in \bar{U}$, Definition 1.1 is equivalent to $f(0, \zeta)=H(0, \zeta)$, for all $\zeta \in \bar{U}$, and $f(U \times \bar{U}) \subset H(U \times \bar{U})$.

(ii) If $H(z, \zeta) \equiv H(z)$ and $f(z, \zeta) \equiv f(z)$, the strong subordination becomes the usual notion of subordination.

As a dual notion of strong differential subordination G.I. Oros has introduced and developed the notion of strong differential superordinations in [19].

Definition $1.2[19]$ Let $f(z, \zeta), H(z, \zeta)$ analytic in $U \times \bar{U}$. The function $f(z, \zeta)$ is said to be strongly superordinate to $H(z, \zeta)$ if there exists a function $w$ analytic in $U$, with $w(0)=0$ and $|w(z)|<1$, such that $H(z, \zeta)=f(w(z), \zeta)$, for all $\zeta \in \bar{U}$. In such a case we write $H(z, \zeta) \prec \prec f(z, \zeta), z \in U, \zeta \in \bar{U}$.

Remark $1.2[19]$ (i) Since $f(z, \zeta)$ is analytic in $U \times \bar{U}$, for all $\zeta \in \bar{U}$, and univalent in $U$, for all $\zeta \in \bar{U}$, Definition 1.2 is equivalent to $H(0, \zeta)=f(0, \zeta)$, for all $\zeta \in \bar{U}$, and $H(U \times \bar{U}) \subset f(U \times \bar{U})$.

(ii) If $H(z, \zeta) \equiv H(z)$ and $f(z, \zeta) \equiv f(z)$, the strong superordination becomes the usual notion of superordination.

Definition 1.3 [1] We denote by $Q^{*}$ the set of functions that are analytic and injective on $\bar{U} \times \bar{U} \backslash E(f, \zeta)$, where $E(f, \zeta)=\left\{y \in \partial U: \lim _{z \rightarrow y} f(z, \zeta)=\infty\right\}$, and are such that $f_{z}^{\prime}(y, \zeta) \neq 0$ for $y \in \partial U \times \bar{U} \backslash E(f, \zeta)$. The subclass of $Q^{*}$ for which $f(0, \zeta)=a$ is denoted by $Q^{*}(a)$.

For two functions $f(z, \zeta)=z+\sum_{j=2}^{\infty} a_{j}(\zeta) z^{j}$ and $g(z, \zeta)=z+\sum_{j=2}^{\infty} b_{j}(\zeta) z^{j}$ analytic in $U \times \bar{U}$, the Hadamard product (or convolution) of $f(z, \zeta)$ and $g(z, \zeta)$, written as $(f * g)(z, \zeta)$ is defined by

$$
f(z, \zeta) * g(z, \zeta)=(f * g)(z, \zeta)=z+\sum_{j=2}^{\infty} a_{j}(\zeta) b_{j}(\zeta) z^{j}
$$

Definition 1.4 ([2]) For $f \in \mathcal{A}_{\zeta}^{*}, \lambda \geq 0$ and $m \in \mathbb{N}$, the extended generalized Sălăgean operator $D_{\lambda}^{m}$ is defined by $D_{\lambda}^{m}: \mathcal{A}_{\zeta}^{*} \rightarrow \mathcal{A}_{\zeta}^{*}$,

$$
\begin{aligned}
D_{\lambda}^{0} f(z, \zeta)= & f(z, \zeta) \\
D_{\lambda}^{1} f(z, \zeta)= & (1-\lambda) f(z, \zeta)+\lambda z f_{z}^{\prime}(z, \zeta)=D_{\lambda} f(z, \zeta) \\
& \cdots \\
D_{\lambda}^{m+1} f(z, \zeta)= & (1-\lambda) D_{\lambda}^{m} f(z, \zeta)+\lambda z\left(D_{\lambda}^{m} f(z, \zeta)\right)_{z}^{\prime}=D_{\lambda}\left(D_{\lambda}^{m} f(z, \zeta)\right),
\end{aligned}
$$


for $z \in U, \zeta \in \bar{U}$.

Remark 1.3 If $f \in \mathcal{A}_{\zeta}^{*}$ and $f(z, \zeta)=z+\sum_{j=2}^{\infty} a_{j}(\zeta) z^{j}$, then

$D_{\lambda}^{m} f(z, \zeta)=z+\sum_{j=2}^{\infty}[1+(j-1) \lambda]^{m} a_{j}(\zeta) z^{j}$, for $z \in U, \zeta \in \bar{U}$.

Definition 1.5 ([3]) For $f \in \mathcal{A}_{\zeta}^{*}, m \in \mathbb{N}$, the extended Ruscheweyh derivative $R^{m}$ is defined by $R^{m}: \mathcal{A}_{\zeta}^{*} \rightarrow \mathcal{A}_{\zeta}^{*}$,

$$
\begin{aligned}
R^{0} f(z, \zeta)= & f(z, \zeta) \\
R^{1} f(z, \zeta)= & z f_{z}^{\prime}(z, \zeta) \\
& \cdots \\
(m+1) R^{m+1} f(z, \zeta)= & z\left(R^{m} f(z, \zeta)\right)_{z}^{\prime}+m R^{m} f(z, \zeta),
\end{aligned}
$$

$z \in U, \zeta \in \bar{U}$.

Remark 1.4 If $f \in \mathcal{A}_{\zeta}^{*}, f(z, \zeta)=z+\sum_{j=2}^{\infty} a_{j}(\zeta) z^{j}$, then $R^{m} f(z, \zeta)=z+$ $\sum_{j=2}^{\infty} \frac{(m+j-1) !}{m !(j-1) !} a_{j}(\zeta) z^{j}, z \in U, \zeta \in \bar{U}$.

In order to prove our strong subordination and strong superordination results, we make use of the following known results.

Lemma 1.1 Let the function $q$ be univalent in $U \times \bar{U}$ and $\theta$ and $\phi$ be analytic in a domain $D$ containing $q(U \times \bar{U})$ with $\phi(w) \neq 0$ when $w \in q(U \times \bar{U})$. Set $Q(z, \zeta)=$ $z q_{z}^{\prime}(z, \zeta) \phi(q(z, \zeta))$ and $h(z, \zeta)=\theta(q(z, \zeta))+Q(z, \zeta)$. Suppose that

1. $Q$ is starlike univalent in $U \times \bar{U}$ and

2. $\operatorname{Re}\left(\frac{z h_{z}^{\prime}(z, \zeta)}{Q(z, \zeta)}\right)>0$ for $z \in U, \zeta \in \bar{U}$.

If $p$ is analytic with $p(0, \zeta)=q(0, \zeta), p(U \times \bar{U}) \subseteq D$ and

$$
\theta(p(z, \zeta))+z p_{z}^{\prime}(z, \zeta) \phi(p(z, \zeta)) \prec \prec \theta(q(z, \zeta))+z q_{z}^{\prime}(z, \zeta) \phi(q(z, \zeta)),
$$

then $p(z, \zeta) \prec \prec q(z, \zeta)$ and $q$ is the best dominant.

Lemma 1.2 Let the function $q$ be convex univalent in $U \times \bar{U}$ and $\nu$ and $\phi$ be analytic in a domain $D$ containing $q(U \times \bar{U})$. Suppose that

1. $\operatorname{Re}\left(\frac{\nu_{z}^{\prime}(q(z, \zeta))}{\phi(q(z, \zeta))}\right)>0$ for $z \in U, \zeta \in \bar{U}$ and

2. $\psi(z, \zeta)=z q_{z}^{\prime}(z, \zeta) \phi(q(z, \zeta))$ is starlike univalent in $U \times \bar{U}$.

If $p(z, \zeta) \in \mathcal{H}^{*}[q(0, \zeta), 1, \zeta] \cap Q^{*}$, with $p(U \times \bar{U}) \subseteq D$ and

$\nu(p(z, \zeta))+z p_{z}^{\prime}(z) \phi(p(z, \zeta))$ is univalent in $U \times \bar{U}$ and

$$
\nu(q(z, \zeta))+z q_{z}^{\prime}(z, \zeta) \phi(q(z, \zeta)) \prec \prec \nu(p(z, \zeta))+z p_{z}^{\prime}(z, \zeta) \phi(p(z, \zeta)),
$$

then $q(z, \zeta) \prec \prec p(z, \zeta)$ and $q$ is the best subordinant. 


\section{Main results}

Extending the results from [11] to the class $\mathcal{A}_{\zeta}^{*}$ we obtain:

Definition 2.1 ([12]) Let $\lambda \geq 0$ and $n, m \in \mathbb{N}$. Denote by $D R_{\lambda}^{m, n}: \mathcal{A}_{\zeta}^{*} \rightarrow \mathcal{A}_{\zeta}^{*}$ the operator given by the Hadamard product of the extended generalized Sălăgean operator $D_{\lambda}^{m}$ and the extended Ruscheweyh operator $R^{n}$,

$$
D R_{\lambda}^{m, n} f(z, \zeta)=\left(D_{\lambda}^{m} * R^{n}\right) f(z, \zeta)
$$

for any $z \in U, \zeta \in \bar{U}$, and each nonnegative integers $m, n$.

Remark 2.1 If $f \in \mathcal{A}_{\zeta}^{*}$ and $f(z, \zeta)=z+\sum_{j=2}^{\infty} a_{j}(\zeta) z^{j}$, then

$$
D R_{\lambda}^{m, n} f(z, \zeta)=z+\sum_{j=2}^{\infty}[1+(j-1) \lambda]^{m} \frac{(n+j-1) !}{n !(j-1) !} a_{j}^{2}(\zeta) z^{j} \text {, for } z \in U, \zeta \in \bar{U} \text {. }
$$

Remark 2.2 For $m=n$ we obtain the operator $D R_{\lambda}^{m}$ studied in [13], [14], [15], [16], [4], [5], [6].

For $\lambda=1, m=n$, we obtain the Hadamard product $S R^{n}$ [7] of the Sălăgean operator $S^{n}$ and Ruscheweyh derivative $R^{n}$, which was studied in [8], [9], [10].

Using simple computation one obtains the next result.

Proposition 2.1 For $m, n \in \mathbb{N}$ and $\lambda \geq 0$ we have For $m, n \in \mathbb{N}$ and $\lambda \geq 0$ we have

$$
D R_{\lambda}^{m+1, n} f(z, \zeta)=(1-\lambda) D R_{\lambda}^{m, n} f(z, \zeta)+\lambda z\left(D R_{\lambda}^{m, n} f(z, \zeta)\right)_{z}^{\prime}
$$

and

$$
z\left(D R_{\lambda}^{m, n} f(z, \zeta)\right)_{z}^{\prime}=(n+1) D R_{\lambda}^{m, n+1} f(z, \zeta)-n D R_{\lambda}^{m, n} f(z, \zeta)
$$

Proof. We have

$$
\begin{aligned}
D R_{\lambda}^{m+1, n} f(z, \zeta)= & z+\sum_{j=2}^{\infty}[1+(j-1) \lambda]^{m+1} \frac{(n+j-1) !}{n !(j-1) !} a_{j}^{2}(\zeta) z^{j} \\
= & z+\sum_{j=2}^{\infty}[(1-\lambda)+\lambda j][1+(j-1) \lambda]^{m} \frac{(n+j-1) !}{n !(j-1) !} a_{j}^{2}(\zeta) z^{j} \\
= & z+(1-\lambda) \sum_{j=2}^{\infty}[1+(j-1) \lambda]^{m} \frac{(n+j-1) !}{n !(j-1) !} a_{j}^{2}(\zeta) z^{j} \\
& +\lambda \sum_{j=2}^{\infty}[1+(j-1) \lambda]^{m} \frac{(n+j-1) !}{n !(j-1) !} j a_{j}^{2}(\zeta) z^{j} \\
= & (1-\lambda) D R_{\lambda}^{m, n} f(z, \zeta)+\lambda z\left(D R_{\lambda}^{m, n} f(z, \zeta)\right)_{z}^{\prime},
\end{aligned}
$$


and

$$
\begin{aligned}
(n+1) & D R_{\lambda}^{m, n+1} f(z, \zeta)-n D R_{\lambda}^{m, n} f(z, \zeta) \\
= & (n+1) z+(n+1) \sum_{j=2}^{\infty}[1+(j-1) \lambda]^{m} \frac{(n+j) !}{(n+1) !(j-1) !} a_{j}^{2}(\zeta) z^{j} \\
& -n z-n \sum_{j=2}^{\infty}[1+(j-1) \lambda]^{m} \frac{(n+j-1) !}{n !(j-1) !} a_{j}^{2}(\zeta) z^{j} \\
= & z+(n+1) \sum_{j=2}^{\infty}[1+(j-1) \lambda]^{m} \frac{n+j}{n+1} \frac{(n+j-1) !}{n !(j-1) !} a_{j}^{2}(\zeta) z^{j} \\
& -n \sum_{j=2}^{\infty}[1+(j-1) \lambda]^{m} \frac{(n+j-1) !}{n !(j-1) !} a_{j}^{2}(\zeta) z^{j} \\
= & z+\sum_{j=2}^{\infty}[1+(j-1) \lambda]^{m} \frac{(n+j-1) !}{n !(j-1) !} j a_{j}^{2}(z) z^{j} \\
= & z\left(D R_{\lambda}^{m, n} f(z, \zeta)\right)_{z}^{\prime} \cdot
\end{aligned}
$$

We begin with the following

Theorem 2.2 Let $\frac{D R_{\lambda}^{m+1, n} f(z, \zeta)}{D R_{\lambda}^{m, n} f(z, \zeta)} \in \mathcal{H}(U \times \bar{U}), z \in U, \zeta \in \bar{U}, f \in \mathcal{A}_{\zeta}^{*}, m, n \in \mathbb{N}, \lambda \geq$ 0 and let the function $q(z, \zeta)$ be convex and univalent in $U \times \bar{U}$ such that $q(0, \zeta)=1$. Assume that

$$
\operatorname{Re}\left(1+\frac{\alpha}{\mu}+\frac{2 \beta}{\mu} q(z, \zeta)+\frac{z q_{z^{2}}^{\prime \prime}(z, \zeta)}{q_{z}^{\prime}(z, \zeta)}\right)>0, \quad z \in U, \zeta \in \bar{U},
$$

for $\alpha, \beta, \mu, \in \mathbb{C}, \mu \neq 0, z \in U, \zeta \in \bar{U}$, and

$$
\begin{aligned}
& \psi_{\lambda}^{m, n}(\alpha, \beta, \mu ; z, \zeta):=\left(\frac{1-\lambda(n+1)}{\lambda} \mu+\alpha\right) \frac{D R_{\lambda}^{m+1, n} f(z, \zeta)}{D R_{\lambda}^{m, n} f(z, \zeta)} \\
& \quad+\mu(n+1)[1-\lambda(n+2)] \frac{D R_{\lambda}^{m, n+1} f(z, \zeta)}{D R_{\lambda}^{m, n} f(z, \zeta)} \\
& \quad+\lambda \mu(n+1)(n+2) \frac{D R_{\lambda}^{m, n+2} f(z, \zeta)}{D R_{\lambda}^{m, n} f(z, \zeta)}+\left(\beta-\frac{\mu}{\lambda}\right)\left(\frac{D R_{\lambda}^{m+1, n} f(z, \zeta)}{D R_{\lambda}^{m, n} f(z, \zeta)}\right)^{2} .
\end{aligned}
$$

If $q$ satisfies the following strong differential subordination

$$
\psi_{\lambda}^{m, n}(\alpha, \beta, \mu ; z, \zeta) \prec \prec \alpha q(z, \zeta)+\beta(q(z, \zeta))^{2}+\mu z q_{z}^{\prime}(z, \zeta),
$$

for, $\alpha, \beta, \mu \in \mathbb{C}, \mu \neq 0$ then

$$
\frac{D R_{\lambda}^{m+1, n} f(z, \zeta)}{D R_{\lambda}^{m, n} f(z, \zeta)} \prec \prec q(z, \zeta), \quad z \in U, \zeta \in \bar{U},
$$


and $q$ is the best dominant.

Proof. Let the function $p$ be defined by $p(z, \zeta):=\frac{D R_{\lambda}^{m+1, n} f(z, \zeta)}{D R_{\lambda}^{m, n} f(z, \zeta)}, z \in U, z \neq 0, \zeta \in \bar{U}$, $f \in \mathcal{A}_{\zeta}^{*}$. The function $p$ is analytic in $U$ and $p(0, \zeta)=1$.

Differentiating with respect to $z$ this function, we get $z p_{z}^{\prime}(z, \zeta)=\frac{z\left(D R_{\lambda}^{m+1, n} f(z, \zeta)\right)_{z}^{\prime}}{D R_{\lambda}^{m, n} f(z, \zeta)}-\frac{D R_{\lambda}^{m+1, n} f(z, \zeta)}{D R_{\lambda}^{m, n} f(z, \zeta)} \frac{z\left(D R_{\lambda}^{m, n} f(z, \zeta)\right)_{z}^{\prime}}{D R_{\lambda}^{m, n} f(z, \zeta)}$

By using the identity (2.1) and (2.2), we obtain

$$
\begin{aligned}
z p_{z}^{\prime}(z, \zeta)= & \frac{1-\lambda(n+1)}{\lambda} \frac{D R_{\lambda}^{m+1, n} f(z, \zeta)}{D R_{\lambda}^{m, n} f(z, \zeta)} \\
& +(n+1)[1-\lambda(n+2)] \frac{D R_{\lambda}^{m, n+1} f(z, \zeta)}{D R_{\lambda}^{m, n} f(z, \zeta)} \\
& +\lambda(n+1)(n+2) \frac{D R_{\lambda}^{m, n+2} f(z, \zeta)}{D R_{\lambda}^{m, n} f(z, \zeta)}-\frac{1}{\lambda}\left(\frac{D R_{\lambda}^{m+1, n} f(z, \zeta)}{D R_{\lambda}^{m, n} f(z, \zeta)}\right)^{2} \\
& +\lambda(n+1)(n+2) \frac{D R_{\lambda}^{m, n+2} f(z, \zeta)}{D R_{\lambda}^{m, n} f(z, \zeta)}-\frac{1}{\lambda}\left(\frac{D R_{\lambda}^{m+1, n} f(z, \zeta)}{D R_{\lambda}^{m, n} f(z, \zeta)}\right)^{2}
\end{aligned}
$$

By setting $\theta(w):=\alpha w+\beta w^{2}$ and $\phi(w):=\mu, \alpha, \beta, \mu \in \mathbb{C}, \mu \neq 0$ it can be easily verified that $\theta$ is analytic in $\mathbb{C}, \phi$ is analytic in $\mathbb{C} \backslash\{0\}$ and that $\phi(w) \neq 0, w \in \mathbb{C} \backslash\{0\}$.

Also, by letting $Q(z, \zeta)=z q_{z}^{\prime}(z, \zeta) \phi(q(z, \zeta))=\mu z q_{z}^{\prime}(z, \zeta)$,we find that $Q(z, \zeta)$ is starlike univalent in $U \times \bar{U}$.

Let $h(z, \zeta)=\theta(q(z, \zeta))+Q(z, \zeta)=\alpha q(z, \zeta)+\beta(q(z, \zeta))^{2}+\mu z q_{z}^{\prime}(z, \zeta), z \in U$, $\zeta \in \bar{U}$.

If we derive the function $Q$, with respect to $z$, perform calculations, we have $\operatorname{Re}\left(\frac{z h_{z}^{\prime}(z, \zeta)}{Q(z, \zeta)}\right)=\operatorname{Re}\left(1+\frac{\alpha}{\mu}+\frac{2 \beta}{\mu} q(z, \zeta)+\frac{z q_{z 2}^{\prime \prime}(z, \zeta)}{q_{z}^{\prime}(z, \zeta)}\right)>0$.

By using (2.7), we obtain $\alpha p(z, \zeta)+\beta(p(z, \zeta))^{2}+\mu z p_{z}^{\prime}(z, \zeta)=$ $\left(\frac{1-\lambda(n+1)}{\lambda} \mu+\alpha\right) \frac{D R_{\lambda}^{m+1, n} f(z, \zeta)}{D R_{\lambda}^{m, n} f(z, \zeta)}+\mu(n+1)[1-\lambda(n+2)] \frac{D R_{\lambda}^{m, n+1} f(z, \zeta)}{D R_{\lambda}^{m, n} f(z, \zeta)}+\lambda \mu(n+1)(n+$ 2) $\frac{D R_{\lambda}^{m, n+2} f(z, \zeta)}{D R_{\lambda}^{m, n} f(z, \zeta)}+\left(\beta-\frac{\mu}{\lambda}\right)\left(\frac{D R_{\lambda}^{m+1, n} f(z, \zeta)}{D R_{\lambda}^{m, n} f(z, \zeta)}\right)^{2}$.

By using (2.5), we have $\alpha p(z, \zeta)+\beta(p(z, \zeta))^{2}+\mu z p_{z}^{\prime}(z, \zeta) \prec \prec \alpha q(z, \zeta)+$ $\beta(q(z, \zeta))^{2}+\mu z q_{z}^{\prime}(z, \zeta)$.

Therefore, the conditions of Lemma 1.1 are met, so we have $p(z, \zeta) \prec \prec q(z, \zeta)$, $z \in U, \zeta \in \bar{U}$, i.e. $\frac{D R_{\lambda}^{m+1, n} f(z, \zeta)}{D R_{\lambda}^{m, n} f(z, \zeta)} \prec \prec q(z, \zeta), z \in U, \zeta \in \bar{U}$, and $q$ is the best dominant.

Corollary 2.3 Let $q(z, \zeta)=\frac{\zeta+A z}{\zeta+B z},-1 \leq B<A \leq 1, m, n \in \mathbb{N}, \lambda \geq 0, z \in U$, $\zeta \in \bar{U}$. Assume that (2.3) holds. If $f \in \mathcal{A}_{\zeta}^{*}$ and

$$
\psi_{\lambda}^{m, n}(\alpha, \beta, \mu ; z, \zeta) \prec \prec \alpha \frac{\zeta+A z}{\zeta+B z}+\beta\left(\frac{\zeta+A z}{\zeta+B z}\right)^{2}+\mu \frac{\zeta(A-B) z}{(\zeta+B z)^{2}},
$$


for $\alpha, \beta, \mu \in \mathbb{C}, \mu \neq 0,-1 \leq B<A \leq 1$, where $\psi_{\lambda}^{m, n}$ is defined in (2.4), then

$$
\frac{D R_{\lambda}^{m+1, n} f(z, \zeta)}{D R_{\lambda}^{m, n} f(z, \zeta)} \prec \prec \frac{\zeta+A z}{\zeta+B z}
$$

and $\frac{\zeta+A z}{\zeta+B z}$ is the best dominant.

Proof. For $q(z, \zeta)=\frac{\zeta+A z}{\zeta+B z},-1 \leq B<A \leq 1$, in Theorem 2.2 we get the corollary.

Corollary 2.4 Let $q(z, \zeta)=\left(\frac{\zeta+z}{\zeta-z}\right)^{\gamma}, m, n \in \mathbb{N}, \lambda \geq 0, z \in U$. Assume that (2.3) holds. If $f \in \mathcal{A}_{\zeta}^{*}$ and

$$
\psi_{\lambda}^{m, n}(\alpha, \beta, \mu ; z, \zeta) \prec \alpha\left(\frac{\zeta+z}{\zeta-z}\right)^{\gamma}+\beta\left(\frac{\zeta+z}{\zeta-z}\right)^{2 \gamma}+\mu \frac{2 \zeta \gamma z}{(\zeta-z)^{2}}\left(\frac{\zeta+z}{\zeta-z}\right)^{\gamma-1}
$$

for $\alpha, \mu, \beta \in \mathbb{C}, 0<\gamma \leq 1, \mu \neq 0$, where $\psi_{\lambda}^{m, n}$ is defined in (2.4), then

$$
\frac{D R_{\lambda}^{m+1, n} f(z, \zeta)}{D R_{\lambda}^{m, n} f(z, \zeta)} \prec \prec\left(\frac{\zeta+z}{\zeta-z}\right)^{\gamma}
$$

and $\left(\frac{\zeta+z}{\zeta-z}\right)^{\gamma}$ is the best dominant.

Proof. Corollary follows by using Theorem 2.2 for $q(z, \zeta)=\left(\frac{\zeta+z}{\zeta-z}\right)^{\gamma}, 0<\gamma \leq 1$.

Theorem 2.5 Let $q$ be convex and univalent in $U \times \bar{U}$, such that $q(0, \zeta)=1, m, n \in$ $\mathbb{N}, \lambda \geq 0$. Assume that

$$
\operatorname{Re}\left(\frac{q_{z}^{\prime}(z, \zeta)}{\mu}(\alpha+2 \beta q(z, \zeta))\right)>0, \text { for } \alpha, \mu, \beta \in \mathbb{C}, \mu \neq 0,
$$

$z \in U, \zeta \in \bar{U}$.

If $f \in \mathcal{A}_{\zeta}^{*}, \frac{D R_{\lambda}^{m+1, n} f(z, \zeta)}{D R_{\lambda}^{m, n} f(z, \zeta)} \in \mathcal{H}^{*}[q(0, \zeta), 1, \zeta] \cap Q^{*}$ and $\psi_{\lambda}^{m, n}(\alpha, \beta, \mu ; z, \zeta)$ is univalent in $U \times \bar{U}$, where $\psi_{\lambda}^{m, n}(\alpha, \beta, \mu ; z, \zeta)$ is as defined in (2.4), then

$$
\alpha q(z, \zeta)+\beta(q(z, \zeta))^{2}+\mu z q_{z}^{\prime}(z, \zeta) \prec \prec \psi_{\lambda}^{m, n}(\alpha, \beta, \mu ; z, \zeta),
$$

$z \in U, \zeta \in \bar{U}$, implies

$$
q(z, \zeta) \prec \prec \frac{D R_{\lambda}^{m+1, n} f(z, \zeta)}{D R_{\lambda}^{m, n} f(z, \zeta)}, \quad z \in U, \zeta \in \bar{U},
$$

and $q$ is the best subordinant. 
Proof. Let the function $p$ be defined by $p(z, \zeta):=\frac{D R_{\lambda}^{m+1, n} f(z, \zeta)}{D R_{\lambda}^{m, n} f(z, \zeta)}, z \in U, z \neq 0$, $\zeta \in \bar{U}, f \in \mathcal{A}_{\zeta}^{*}$.

By setting $\nu(w):=\alpha w+\beta w^{2}$ and $\phi(w):=\mu$ it can be easily verified that $\nu$ is analytic in $\mathbb{C}, \phi$ is analytic in $\mathbb{C} \backslash\{0\}$ and that $\phi(w) \neq 0, w \in \mathbb{C} \backslash\{0\}$.

Since $\frac{\nu_{z}^{\prime}(q(z, \zeta))}{\phi(q(z, \zeta))}=\frac{q_{z}^{\prime}(z, \zeta)}{\mu}(\alpha+2 \beta q(z, \zeta))$, it follows that

$$
\operatorname{Re}\left(\frac{\nu_{z}^{\prime}(q(z, \zeta))}{\phi(q(z, \zeta))}\right)=\operatorname{Re}\left(\frac{q_{z}^{\prime}(z, \zeta)}{\mu}(\alpha+2 \beta q(z, \zeta))\right)>0
$$

for $\mu, \xi, \beta \in \mathbb{C}, \mu \neq 0$.

By using (2.9) we obtain

$$
\begin{gathered}
\alpha q(z, \zeta)+\beta(q(z, \zeta))^{2}+\mu z q_{z}^{\prime}(z, \zeta) \prec \prec \\
\alpha q(z, \zeta)+\beta(q(z, \zeta))^{2}+\mu z q_{z}^{\prime}(z, \zeta) .
\end{gathered}
$$

Using Lemma 1.2, we have

$$
q(z, \zeta) \prec \prec p(z, \zeta)=\frac{D R_{\lambda}^{m+1, n} f(z, \zeta)}{D R_{\lambda}^{m, n} f(z, \zeta)}, \quad z \in U, \zeta \in \bar{U},
$$

and $q$ is the best subordinant.

Corollary 2.6 Let $q(z, \zeta)=\frac{\zeta+A z}{\zeta+B z},-1 \leq B<A \leq 1, m, n \in \mathbb{N}, \lambda \geq 0$. Assume that (2.8) holds.

$$
\begin{aligned}
\text { If } f \in \mathcal{A}_{\zeta}^{*}, \frac{D R_{\lambda}^{m+1, n} f(z, \zeta)}{D R_{\lambda}^{m, n} f(z, \zeta)} \in \mathcal{H}^{*}[q(0, \zeta), 1, \zeta] \cap Q^{*} \text { and } \\
\\
\alpha \frac{\zeta+A z}{\zeta+B z}+\beta\left(\frac{\zeta+A z}{\zeta+B z}\right)^{2}+\mu \frac{\zeta(A-B) z}{(\zeta+B z)^{2}} \prec \prec \psi_{\lambda}^{m, n}(\alpha, \beta, \mu ; z, \zeta),
\end{aligned}
$$

for $\alpha, \mu, \beta \in \mathbb{C}, \mu \neq 0,-1 \leq B<A \leq 1$, where $\psi_{\lambda}^{m, n}$ is defined in (2.4), then

$$
\frac{\zeta+A z}{\zeta+B z} \prec \prec \frac{D R_{\lambda}^{m+1, n} f(z, \zeta)}{D R_{\lambda}^{m, n} f(z, \zeta)}
$$

and $\frac{\zeta+A z}{\zeta+B z}$ is the best subordinant.

Proof. For $q(z, \zeta)=\frac{\zeta+A z}{\zeta+B z},-1 \leq B<A \leq 1$ in Theorem 2.5 we get the corollary.

Corollary 2.7 Let $q(z, \zeta)=\left(\frac{\zeta+z}{\zeta-z}\right)^{\gamma}, m, n \in \mathbb{N}, \lambda \geq 0$. Assume that (2.8) holds.

If $f \in \mathcal{A}_{\zeta}^{*}, \frac{D R_{\lambda}^{m+1, n} f(z, \zeta)}{D R_{\lambda}^{m, n} f(z, \zeta)} \in \mathcal{H}^{*}[q(0, \zeta), 1, \zeta] \cap Q^{*}$ and

$$
\begin{aligned}
\alpha\left(\frac{\zeta+z}{\zeta-z}\right)^{\gamma}+ & \beta\left(\frac{\zeta+z}{\zeta-z}\right)^{2 \gamma}+\mu \frac{2 \zeta \gamma z}{(\zeta-z)^{2}}\left(\frac{\zeta+z}{\zeta-z}\right)^{\gamma-1} \\
& \prec \prec \psi_{\lambda}^{m, n}(\alpha, \beta, \mu ; z, \zeta),
\end{aligned}
$$


for $\alpha, \mu, \beta \in \mathbb{C}, 0<\gamma \leq 1, \mu \neq 0$, where $\psi_{\lambda}^{m, n}$ is defined in (2.4), then

$$
\left(\frac{\zeta+z}{\zeta-z}\right)^{\gamma} \prec \prec \frac{D R_{\lambda}^{m+1, n} f(z, \zeta)}{D R_{\lambda}^{m, n} f(z, \zeta)}
$$

and $\left(\frac{\zeta+z}{\zeta-z}\right)^{\gamma}$ is the best subordinant.

Proof. Corollary follows by using Theorem 2.5 for $q(z, \zeta)=\left(\frac{\zeta+z}{\zeta-z}\right)^{\gamma}, 0<\gamma \leq 1$.

Combining Theorem 2.2 and Theorem 2.5, we state the following sandwich theorem.

Theorem 2.8 Let $q_{1}$ and $q_{2}$ be analytic and univalent in $U \times \bar{U}$ such that $q_{1}(z, \zeta) \neq 0$ and $q_{2}(z, \zeta) \neq 0$, for all $z \in U, \zeta \in \bar{U}$, with $z\left(q_{1}\right)_{z}^{\prime}(z, \zeta)$ and $z\left(q_{2}\right)_{z}^{\prime}(z, \zeta)$ being starlike univalent. Suppose that $q_{1}$ satisfies (2.3) and $q_{2}$ satisfies (2.8). If $f \in \mathcal{A}_{\zeta}^{*}$, $\frac{D R_{\lambda}^{m+1, n} f(z, \zeta)}{D R_{\lambda}^{m, n} f(z, \zeta)} \in \mathcal{H}^{*}[q(0, \zeta), 1, \zeta] \cap Q^{*}$ and $\psi_{\lambda}^{m, n}(\alpha, \beta, \mu ; z, \zeta)$ is as defined in (2.4) univalent in $U \times \bar{U}$, then

$$
\begin{aligned}
\alpha q_{1}(z, \zeta) & +\beta\left(q_{1}(z, \zeta)\right)^{2}+\mu z\left(q_{1}\right)_{z}^{\prime}(z, \zeta) \prec \prec \psi_{\lambda}^{m, n}(\alpha, \beta, \mu ; z, \zeta) \\
& \prec \prec \alpha q_{2}(z, \zeta)+\beta\left(q_{2}(z, \zeta)\right)^{2}+\mu z\left(q_{2}\right)_{z}^{\prime}(z, \zeta),
\end{aligned}
$$

for $\alpha, \mu, \beta \in \mathbb{C}, \mu \neq 0$, implies

$$
q_{1}(z, \zeta) \prec \prec \frac{D R_{\lambda}^{m+1, n} f(z, \zeta)}{D R_{\lambda}^{m, n} f(z, \zeta)} \prec \prec q_{2}(z, \zeta), \quad \delta \in \mathbb{C}, \delta \neq 0,
$$

and $q_{1}$ and $q_{2}$ are respectively the best subordinant and the best dominant.

For $q_{1}(z, \zeta)=\frac{\zeta+A_{1} z}{\zeta+B_{1} z}, q_{2}(z, \zeta)=\frac{\zeta+A_{2} z}{\zeta+B_{2} z}$, where $-1 \leq B_{2}<B_{1}<A_{1}<A_{2} \leq 1$, we have the following corollary.

Corollary 2.9 Let $m, n \in \mathbb{N}, \lambda \geq 0$. Assume that (2.3) and (2.8) hold for $q_{1}(z, \zeta)=\frac{\zeta+A_{1} z}{\zeta+B_{1} z}$ and $q_{2}(z, \zeta)=\frac{\zeta+A_{2} z}{\zeta+B_{2} z}$, respectively. If $f \in \mathcal{A}_{\zeta}^{*}, \frac{D R_{\lambda}^{m+1, n} f(z, \zeta)}{D R_{\lambda}^{m, n} f(z, \zeta)} \in$ $\mathcal{H}^{*}[q(0, \zeta), 1, \zeta] \cap Q^{*}$ and

$$
\begin{aligned}
& \alpha \frac{\zeta+A_{1} z}{\zeta+B_{1} z}+\beta\left(\frac{\zeta+A_{1} z}{\zeta+B_{1} z}\right)^{2}+\mu \frac{\left(A_{1}-B_{1}\right) \zeta z}{\left(\zeta+B_{1} z\right)^{2}} \prec \prec \psi_{\lambda}^{m, n}(\alpha, \beta, \mu ; z, \zeta) \\
& \prec \prec \alpha \frac{\zeta+A_{2} z}{\zeta+B_{2} z}+\beta\left(\frac{\zeta+A_{2} z}{\zeta+B_{2} z}\right)^{2}+\mu \frac{\left(A_{2}-B_{2}\right) \zeta z}{\left(\zeta+B_{2} z\right)^{2}},
\end{aligned}
$$

for $\alpha, \mu, \beta \in \mathbb{C}, \mu \neq 0,-1 \leq B_{2} \leq B_{1}<A_{1} \leq A_{2} \leq 1$, where $\psi_{\lambda}^{m, n}$ is defined in (2.4), then

$$
\frac{\zeta+A_{1} z}{\zeta+B_{1} z} \prec \prec \frac{D R_{\lambda}^{m+1, n} f(z, \zeta)}{D R_{\lambda}^{m, n} f(z, \zeta)} \prec \prec \frac{\zeta+A_{2} z}{\zeta+B_{2} z},
$$

hence $\frac{\zeta+A_{1} z}{\zeta+B_{1} z}$ and $\frac{\zeta+A_{2} z}{\zeta+B_{2} z}$ are the best subordinant and the best dominant, respectively. 
Theorem 2.10 Let $\left(\frac{D R_{\lambda}^{m+1, n} f(z, \zeta)}{D R_{\lambda}^{m, n} f(z, \zeta)}\right)^{\delta} \in \mathcal{H}(U \times \bar{U}), f \in \mathcal{A}_{\zeta}^{*}, z \in U, \zeta \in \bar{U}, \delta \in \mathbb{C}$, $\delta \neq 0, m, n \in \mathbb{N}, \lambda \geq 0$ and let the function $q(z, \zeta)$ be convex and univalent in $U \times \bar{U}$ such that $q(0, \zeta)=1, \zeta \in \bar{U}$. Assume that

$$
\operatorname{Re}\left(\frac{\alpha+\beta}{\beta}+\frac{z q_{z^{2}}^{\prime \prime}(z, \zeta)}{q_{z}^{\prime}(z, \zeta)}\right)>0
$$

for $\alpha, \beta \in \mathbb{C}, \beta \neq 0, z \in U, \zeta \in \bar{U}$, and

$$
\begin{aligned}
& \psi_{\lambda}^{m, n}(\alpha, \beta ; z, \zeta):=\left(\frac{D R_{\lambda}^{m+1, n} f(z, \zeta)}{D R_{\lambda}^{m, n} f(z, \zeta)}\right)^{\delta} \\
& \cdot\left[\alpha+\delta \beta \frac{1-\lambda(n+1)}{\lambda}+\delta \beta(n+1)[1-\lambda(n+2)] \frac{D R_{\lambda}^{m, n+1} f(z, \zeta)}{D R_{\lambda}^{m+1, n} f(z, \zeta)}\right. \\
& \left.\quad+\delta \beta \lambda(n+1)(n+2) \frac{D R_{\lambda}^{m, n+2} f(z, \zeta)}{D R_{\lambda}^{m+1, n} f(z, \zeta)}-\frac{\delta \beta}{\lambda} \frac{D R_{\lambda}^{m+1, n} f(z, \zeta)}{D R_{\lambda}^{m, n} f(z, \zeta)}\right]
\end{aligned}
$$

If $q$ satisfies the following strong differential subordination

$$
\psi_{\lambda}^{m, n}(\alpha, \beta ; z, \zeta) \prec \prec \alpha q(z, \zeta)+\beta z q_{z}^{\prime}(z, \zeta),
$$

for $\alpha, \beta \in \mathbb{C}, \beta \neq 0, z \in U, \zeta \in \bar{U}$, then

$$
\left(\frac{D R_{\lambda}^{m+1, n} f(z, \zeta)}{D R_{\lambda}^{m, n} f(z, \zeta)}\right)^{\delta} \prec \prec q(z, \zeta), \quad z \in U, \zeta \in \bar{U}, \delta \in \mathbb{C}, \delta \neq 0,
$$

and $q$ is the best dominant.

Proof. Let the function $p$ be defined by $p(z, \zeta):=\left(\frac{D R_{\lambda}^{m+1, n} f(z, \zeta)}{D R_{\lambda}^{m, n} f(z, \zeta)}\right)^{\delta}, z \in U$, $z \neq 0, \zeta \in \bar{U}, f \in \mathcal{A}_{\zeta}^{*}$. The function $p$ is analytic in $U \times \bar{U}$ and $p(0, \zeta)=1$.

We have

$$
\begin{aligned}
z p_{z}^{\prime}(z, \zeta)= & \delta z\left(\frac{D R_{\lambda}^{m+1, n} f(z, \zeta)}{D R_{\lambda}^{m, n} f(z, \zeta)}\right)^{\delta} \frac{D R_{\lambda}^{m, n} f(z, \zeta)}{D R_{\lambda}^{m+1, n} f(z, \zeta)}\left(\frac{D R_{\lambda}^{m+1, n} f(z, \zeta)}{D R_{\lambda}^{m, n} f(z, \zeta)}\right)_{z}^{\prime} \\
= & \delta\left(\frac{D R_{\lambda}^{m+1, n} f(z, \zeta)}{D R_{\lambda}^{m, n} f(z, \zeta)}\right)^{\delta} \frac{D R_{\lambda}^{m, n} f(z, \zeta)}{D R_{\lambda}^{m+1, n} f(z, \zeta)} \\
& \cdot\left(\frac{z\left(D R_{\lambda}^{m+1, n} f(z, \zeta)\right)_{z}^{\prime}}{D R_{\lambda}^{m, n} f(z, \zeta)}-\frac{D R_{\lambda}^{m+1, n} f(z, \zeta)}{D R_{\lambda}^{m, n} f(z, \zeta)} \frac{z\left(D R_{\lambda}^{m, n} f(z, \zeta)\right)_{z}^{\prime}}{D R_{\lambda}^{m, n} f(z, \zeta)}\right)
\end{aligned}
$$


By using the identity (2.1) and (2.2), we obtain

$$
\begin{aligned}
z p_{z}^{\prime}(z, \zeta)= & \delta\left(\frac{D R_{\lambda}^{m+1, n} f(z, \zeta)}{D R_{\lambda}^{m, n} f(z, \zeta)}\right)^{\delta} \frac{D R_{\lambda}^{m, n} f(z, \zeta)}{D R_{\lambda}^{m+1, n} f(z, \zeta)} \\
& \cdot\left[\left(\frac{1-\lambda(n+1)}{\lambda}\right) \frac{D R_{\lambda}^{m+1, n} f(z, \zeta)}{D R_{\lambda}^{m, n} f(z, \zeta)}+n+1\right) \\
& \cdot[1-\lambda(n+2)] \frac{D R_{\lambda}^{m, n+1} f(z, \zeta)}{D R_{\lambda}^{m, n} f(z, \zeta)}+\lambda(n+1)(n+2) \frac{D R_{\lambda}^{m, n+2} f(z, \zeta)}{D R_{\lambda}^{m, n} f(z, \zeta)} \\
& \left.-\frac{1}{\lambda}\left(\frac{D R_{\lambda}^{m+1, n} f(z, \zeta)}{D R_{\lambda}^{m, n} f(z, \zeta)}\right)^{2}\right]
\end{aligned}
$$

so, we obtain

$$
\begin{gathered}
z p_{z}^{\prime}(z, \zeta)=\delta\left(\frac{D R_{\lambda}^{m+1, n} f(z, \zeta)}{D R_{\lambda}^{m, n} f(z, \zeta)}\right)^{\delta}\left[\frac{1-\lambda(n+1)}{\lambda}+\right. \\
(n+1)[1-\lambda(n+2)] \frac{D R_{\lambda}^{m, n+1} f(z, \zeta)}{D R_{\lambda}^{m+1, n} f(z, \zeta)}+ \\
\left.\lambda(n+1)(n+2) \frac{D R_{\lambda}^{m, n+2} f(z, \zeta)}{D R_{\lambda}^{m+1, n} f(z, \zeta)}-\frac{1}{\lambda} \frac{D R_{\lambda}^{m+1, n} f(z, \zeta)}{D R_{\lambda}^{m, n} f(z, \zeta)}\right]
\end{gathered}
$$

By setting $\theta(w):=\alpha w$ and $\phi(w):=\beta$, it can be easily verified that $\theta$ is analytic in $\mathbb{C}, \phi$ is analytic in $\mathbb{C} \backslash\{0\}$ and that $\phi(w) \neq 0, w \in \mathbb{C} \backslash\{0\}$.

Also, by letting $Q(z, \zeta)=z q_{z}^{\prime}(z, \zeta) \phi(q(z, \zeta))=\beta z q_{z}^{\prime}(z, \zeta)$, we find that $Q(z, \zeta)$ is starlike univalent in $U \times \bar{U}$.

Let $h(z, \zeta)=\theta(q(z, \zeta))+Q(z, \zeta)=\alpha q(z, \zeta)+\beta z q_{z}^{\prime}(z, \zeta)$.

We have $\operatorname{Re}\left(\frac{z h_{z}^{\prime}(z, \zeta)}{Q(z, \zeta)}\right)=\operatorname{Re}\left(\frac{\alpha+\beta}{\beta}+\frac{z q_{z}^{\prime \prime}(z, \zeta)}{q_{z}^{\prime}(z, \zeta)}\right)>0$.

By using (2.16), we obtain

$$
\begin{aligned}
& \alpha p(z, \zeta)+\beta z p_{z}^{\prime}(z, \zeta)=\left(\frac{D R_{\lambda}^{m+1, n} f(z, \zeta)}{D R_{\lambda}^{m, n} f(z, \zeta)}\right)^{\delta} \\
& \cdot\left[\alpha+\delta \beta \frac{1-\lambda(n+1)}{\lambda}+\delta \beta(n+1)[1-\lambda(n+2)] \frac{D R_{\lambda}^{m, n+1} f(z, \zeta)}{D R_{\lambda}^{m+1, n} f(z, \zeta)}\right. \\
& \left.+\delta \beta \lambda(n+1)(n+2) \frac{D R_{\lambda}^{m, n+2} f(z, \zeta)}{D R_{\lambda}^{m+1, n} f(z, \zeta)}-\frac{\delta \beta}{\lambda} \frac{D R_{\lambda}^{m+1, n} f(z, \zeta)}{D R_{\lambda}^{m, n} f(z, \zeta)}\right] .
\end{aligned}
$$

By using (2.13), we have $\alpha p(z, \zeta)+\beta z p_{z}^{\prime}(z, \zeta) \prec \prec \alpha q(z, \zeta)+\beta z q_{z}^{\prime}(z, \zeta)$.

From Lemma 1.1, we have $p(z, \zeta) \prec \prec q(z, \zeta), z \in U, \zeta \in \bar{U}$, i.e. $\left(\frac{D R_{\lambda}^{m+1, n} f(z, \zeta)}{D R_{\lambda}^{m, n} f(z, \zeta)}\right)^{\delta} \prec \prec$ $q(z, \zeta), z \in U, \zeta \in \bar{U}, \delta \in \mathbb{C}, \delta \neq 0$ and $q$ is the best dominant. 
Corollary 2.11 Let $q(z, \zeta)=\frac{\zeta+A z}{\zeta+B z}, z \in U, \zeta \in \bar{U},-1 \leq B<A \leq 1, m, n \in \mathbb{N}$, $\lambda \geq 0$. Assume that (2.11) holds. If $f \in \mathcal{A}_{\zeta}^{*}$ and

$$
\psi_{\lambda}^{m, n}(\alpha, \beta ; z, \zeta) \prec \prec \alpha \frac{\zeta+A z}{\zeta+B z}+\beta \frac{(A-B) \zeta z}{(\zeta+B z)^{2}},
$$

for $\alpha, \beta \in \mathbb{C}, \beta \neq 0,-1 \leq B<A \leq 1$, where $\psi_{\lambda}^{m, n}$ is defined in (2.12), then

$$
\left(\frac{D R_{\lambda}^{m+1, n} f(z, \zeta)}{D R_{\lambda}^{m, n} f(z, \zeta)}\right)^{\delta} \prec \prec \frac{\zeta+A z}{\zeta+B z}, \quad \delta \in \mathbb{C}, \delta \neq 0,
$$

and $\frac{\zeta+A z}{\zeta+B z}$ is the best dominant.

Proof. For $q(z, \zeta)=\frac{\zeta+A z}{\zeta+B z},-1 \leq B<A \leq 1$, in Theorem 2.10 we get the corollary.

Corollary 2.12 Let $q(z, \zeta)=\left(\frac{\zeta+z}{\zeta-z}\right)^{\gamma}, m, n \in \mathbb{N}, \lambda \geq 0$. Assume that (2.11) holds. If $f \in \mathcal{A}_{\zeta}^{*}$ and

$$
\psi_{\lambda}^{m, n}(\alpha, \beta, \mu ; z, \zeta) \prec \prec \alpha\left(\frac{\zeta+z}{\zeta-z}\right)^{\gamma}+\beta \frac{2 \gamma \zeta z}{(\zeta-z)^{2}}\left(\frac{\zeta+z}{\zeta-z}\right)^{\gamma-1},
$$

for $\alpha, \beta \in \mathbb{C}, 0<\gamma \leq 1, \beta \neq 0$, where $\psi_{\lambda}^{m, n}$ is defined in (2.12), then

$$
\left(\frac{D R_{\lambda}^{m+1, n} f(z, \zeta)}{D R_{\lambda}^{m, n} f(z, \zeta)}\right)^{\delta} \prec \prec\left(\frac{\zeta+z}{\zeta-z}\right)^{\gamma}, \quad \delta \in \mathbb{C}, \delta \neq 0,
$$

and $\left(\frac{\zeta+z}{\zeta-z}\right)^{\gamma}$ is the best dominant.

Proof. Corollary follows by using Theorem 2.10 for $q(z, \zeta)=\left(\frac{\zeta+z}{\zeta-z}\right)^{\gamma}, 0<\gamma \leq 1$.

Theorem 2.13 Let $q$ be convex and univalent in $U \times \bar{U}$ such that $q(0, \zeta)=1$. Assume that

$$
\operatorname{Re}\left(\frac{\alpha}{\beta} q_{z}^{\prime}(z, \zeta)\right)>0, \text { for } \alpha, \beta \in \mathbb{C}, \beta \neq 0 .
$$

If $f \in \mathcal{A}_{\zeta}^{*},\left(\frac{D R_{\lambda}^{m+1, n} f(z, \zeta)}{D R_{\lambda}^{m, n} f(z, \zeta)}\right)^{\delta} \in \mathcal{H}^{*}[q(0, \zeta), 1, \zeta] \cap Q^{*}$ and $\psi_{\lambda}^{m, n}(\alpha, \beta ; z, \zeta)$ is univalent in $U \times \bar{U}$, where $\psi_{\lambda}^{m, n}(\alpha, \beta ; z, \zeta)$ is as defined in (2.12), then

$$
\alpha q(z, \zeta)+\beta z q_{z}^{\prime}(z, \zeta) \prec \prec \psi_{\lambda}^{m, n}(\alpha, \beta ; z, \zeta)
$$

implies

$$
q(z, \zeta) \prec \prec\left(\frac{D R_{\lambda}^{m+1, n} f(z, \zeta)}{D R_{\lambda}^{m, n} f(z, \zeta)}\right)^{\delta}, \quad \delta \in \mathbb{C}, \delta \neq 0, z \in U, \zeta \in \bar{U},
$$

and $q$ is the best subordinant. 
Proof. Let the function $p$ be defined by $p(z, \zeta):=\left(\frac{D R_{\lambda}^{m+1, n} f(z, \zeta)}{D R_{\lambda}^{m, n} f(z, \zeta)}\right)^{\delta}, z \in U$, $z \neq 0, \zeta \in \bar{U}, \delta \in \mathbb{C}, \delta \neq 0, f \in \mathcal{A}_{\zeta}^{*}$. The function $p$ is analytic in $U \times \bar{U}$ and $p(0, \zeta)=1$.

By setting $\nu(w):=\alpha w$ and $\phi(w):=\beta$ it can be easily verified that $\nu$ is analytic in $\mathbb{C}, \phi$ is analytic in $\mathbb{C} \backslash\{0\}$ and that $\phi(w) \neq 0, w \in \mathbb{C} \backslash\{0\}$.

Since $\frac{\nu_{z}^{\prime}(q(z, \zeta))}{\phi(q(z, \zeta))}=\frac{\alpha}{\beta} q_{z}^{\prime}(z, \zeta)$, it follows that $\operatorname{Re}\left(\frac{\nu_{z}^{\prime}(q(z, \zeta))}{\phi(q(z, \zeta))}\right)=\operatorname{Re}\left(\frac{\alpha}{\beta} q_{z}^{\prime}(z, \zeta)\right)>0$, for $\alpha, \beta \in \mathbb{C}, \beta \neq 0$.

Now, by using (2.18) we obtain

$$
\alpha q(z, \zeta)+\beta z q_{z}^{\prime}(z, \zeta) \prec \prec \alpha q(z, \zeta)+\beta z q_{z}^{\prime}(z, \zeta), \quad z \in U, \zeta \in \bar{U} .
$$

From Lemma 1.2, we have

$$
q(z, \zeta) \prec \prec p(z, \zeta)=\left(\frac{D R_{\lambda}^{m+1, n} f(z, \zeta)}{D R_{\lambda}^{m, n} f(z, \zeta)}\right)^{\delta}
$$

$z \in U, \zeta \in \bar{U}, \delta \in \mathbb{C}, \delta \neq 0$, and $q$ is the best subordinant.

Corollary 2.14 Let $q(z, \zeta)=\frac{\zeta+A z}{\zeta+B z},-1 \leq B<A \leq 1, z \in U, \zeta \in \bar{U}, m, n \in \mathbb{N}$, $\lambda \geq 0$. Assume that (2.17) holds. If $f \in \mathcal{A}_{\zeta}^{*},\left(\frac{D R_{\lambda}^{m+1, n} f(z, \zeta)}{D R_{\lambda}^{m, n} f(z, \zeta)}\right)^{\delta} \in \mathcal{H}^{*}[q(0, \zeta), 1, \zeta] \cap Q^{*}$, $\delta \in \mathbb{C}, \delta \neq 0$ and

$$
\alpha \frac{\zeta+A z}{\zeta+B z}+\beta \frac{(A-B) \zeta z}{(\zeta+B z)^{2}} \prec \prec \psi_{\lambda}^{m, n}(\alpha, \beta ; z, \zeta)
$$

for $\alpha, \beta \in \mathbb{C}, \beta \neq 0,-1 \leq B<A \leq 1$, where $\psi_{\lambda}^{m, n}$ is defined in (2.12), then

$$
\frac{\zeta+A z}{\zeta+B z} \prec \prec\left(\frac{D R_{\lambda}^{m+1, n} f(z, \zeta)}{D R_{\lambda}^{m, n} f(z, \zeta)}\right)^{\delta}, \delta \in \mathbb{C}, \delta \neq 0,
$$

and $\frac{\zeta+A z}{\zeta+B z}$ is the best subordinant.

Proof. For $q(z, \zeta)=\frac{\zeta+A z}{\zeta+B z},-1 \leq B<A \leq 1$, in Theorem 2.13 we get the corollary.

Corollary 2.15 Let $q(z, \zeta)=\left(\frac{\zeta+z}{\zeta-z}\right)^{\gamma}, m, n \in \mathbb{N}, \lambda \geq 0$. Assume that (2.17) holds. If $f \in \mathcal{A}_{\zeta}^{*},\left(\frac{D R_{\lambda}^{m+1, n} f(z, \zeta)}{D R_{\lambda}^{m, n} f(z, \zeta)}\right)^{\delta} \in \mathcal{H}^{*}[q(0, \zeta), 1, \zeta] \cap Q^{*}$ and

$$
\alpha\left(\frac{\zeta+z}{\zeta-z}\right)^{\gamma}+\beta \frac{2 \gamma \zeta z}{(\zeta-z)^{2}}\left(\frac{\zeta+z}{\zeta-z}\right)^{\gamma-1} \prec \prec \psi_{\lambda}^{m, n}(\alpha, \beta, \mu ; z, \zeta),
$$


for $\alpha, \beta \in \mathbb{C}, 0<\gamma \leq 1, \beta \neq 0$, where $\psi_{\lambda}^{m, n}$ is defined in (2.12), then

$$
\left(\frac{\zeta+z}{\zeta-z}\right)^{\gamma} \prec \prec\left(\frac{D R_{\lambda}^{m+1, n} f(z, \zeta)}{D R_{\lambda}^{m, n} f(z, \zeta)}\right)^{\delta}, \quad \delta \in \mathbb{C}, \delta \neq 0,
$$

and $\left(\frac{\zeta+z}{\zeta-z}\right)^{\gamma}$ is the best subordinant.

Proof. Corollary follows by using Theorem 2.13 for $q(z, \zeta)=\left(\frac{\zeta+z}{\zeta-z}\right)^{\gamma}, 0<\gamma \leq 1$.

Combining Theorem 2.10 and Theorem 2.13, we state the following sandwich theorem.

Theorem 2.16 Let $q_{1}$ and $q_{2}$ be convex and univalent in $U \times \bar{U}$ such that $q_{1}(z, \zeta) \neq 0$ and $q_{2}(z, \zeta) \neq 0$, for all $z \in U, \zeta \in \bar{U}$. Suppose that $q_{1}$ satisfies (2.11) and $q_{2}$ satisfies (2.17). If $f \in \mathcal{A}_{\zeta}^{*},\left(\frac{D R_{\lambda}^{m+1, n} f(z, \zeta)}{D R_{\lambda}^{m, n} f(z, \zeta)}\right)^{\delta} \in \mathcal{H}^{*}[q(0, \zeta), 1, \zeta] \cap Q^{*}, \delta \in \mathbb{C}, \delta \neq 0$ and $\psi_{\lambda}^{m, n}(\alpha, \beta ; z, \zeta)$ is as defined in (2.12) univalent in $U \times \bar{U}$, then

$$
\begin{gathered}
\alpha q_{1}(z, \zeta)+\beta z\left(q_{1}\right)_{z}^{\prime}(z, \zeta) \prec \prec \psi_{\lambda}^{m, n}(\alpha, \beta ; z, \zeta) \\
\prec \prec \alpha q_{2}(z, \zeta)+\beta z\left(q_{2}\right)_{z}^{\prime}(z, \zeta),
\end{gathered}
$$

for $\alpha, \beta \in \mathbb{C}, \beta \neq 0$, implies

$$
q_{1}(z, \zeta) \prec \prec\left(\frac{D R_{\lambda}^{m+1, n} f(z, \zeta)}{D R_{\lambda}^{m, n} f(z, \zeta)}\right)^{\delta} \prec \prec q_{2}(z, \zeta),
$$

$z \in U, \zeta \in \bar{U}, \delta \in \mathbb{C}, \delta \neq 0$, and $q_{1}$ and $q_{2}$ are respectively the best subordinant and the best dominant.

For $q_{1}(z, \zeta)=\frac{\zeta+A_{1} z}{\zeta+B_{1} z}, q_{2}(z, \zeta)=\frac{\zeta+A_{2} z}{\zeta+B_{2} z}$, where $-1 \leq B_{2}<B_{1}<A_{1}<A_{2} \leq 1$, we have the following corollary.

Corollary 2.17 Let $m, n \in \mathbb{N}, \lambda \geq 0$. Assume that (2.11) and (2.17) hold for $q_{1}(z, \zeta)=\frac{\zeta+A_{1} z}{\zeta+B_{1} z}$ and $q_{2}(z, \zeta)=\frac{\zeta+A_{2} z}{\zeta+B_{2} z}$, respectively. If $f \in \mathcal{A}_{\zeta}^{*},\left(\frac{D R_{\lambda}^{m+1, n} f(z, \zeta)}{D R_{\lambda}^{m, n} f(z, \zeta)}\right)^{\delta} \in$ $\mathcal{H}^{*}[q(0, \zeta), 1, \zeta] \cap Q^{*}$ and

$$
\begin{aligned}
& \alpha \frac{\zeta+A_{1} z}{\zeta+B_{1} z}+\beta \frac{\left(A_{1}-B_{1}\right) \zeta z}{\left(\zeta+B_{1} z\right)^{2}} \prec \prec \psi_{\lambda}^{m, n}(\alpha, \beta, \mu ; z, \zeta) \\
& \prec \prec \alpha \frac{\zeta+A_{2} z}{\zeta+B_{2} z}+\beta \frac{\left(A_{2}-B_{2}\right) \zeta z}{\left(\zeta+B_{2} z\right)^{2}}, \quad z \in U, \quad \zeta \in \bar{U},
\end{aligned}
$$

for $\alpha, \beta \in \mathbb{C}, \beta \neq 0,-1 \leq B_{2} \leq B_{1}<A_{1} \leq A_{2} \leq 1$, where $\psi_{\lambda}^{m, n}$ is defined in (2.4), then

$$
\frac{\zeta+A_{1} z}{\zeta+B_{1} z} \prec \prec\left(\frac{D R_{\lambda}^{m+1, n} f(z, \zeta)}{D R_{\lambda}^{m, n} f(z, \zeta)}\right)^{\delta} \prec \prec \frac{\zeta+A_{2} z}{\zeta+B_{2} z},
$$

$z \in U, \zeta \in \bar{U}, \delta \in \mathbb{C}, \delta \neq 0$, hence $\frac{\zeta+A_{1} z}{\zeta+B_{1} z}$ and $\frac{\zeta+A_{2} z}{\zeta+B_{2} z}$ are the best subordinant and the best dominant, respectively. 


\section{References}

[1] A. Alb Lupaş, On special strong differential superordinations using Sălăgean and Ruscheweyh operators, submitted Jokull Journal.

[2] A. Alb Lupaş, On special strong differential subordinations using a generalized Sălăgean operator and Ruscheweyh derivative, Journal of Concrete and Applicable Mathematics, Vol. 10, No.'s 1-2, 2012, 17-23.

[3] A. Alb Lupaş, G.I. Oros, Gh. Oros, On special strong differential subordinations using Sălăgean and Ruscheweyh operators, Journal of Computational Analysis and Applications, Vol. 14, No. 2, 2012, 266-270.

[4] A. Alb Lupas,, A note on strong differential subordinations using a generalized Sălăgean operator and Ruscheweyh operator, Acta Universitatis Apulensis No. 34/2013, 105-114.

[5] A. Alb Lupaş, Certain strong differential superordinations using a generalized Sălăgean operator and Ruscheweyh operator, Journal of Applied Functional Analysis, Vol. 7, No.'s 1-2, 2012, 62-68.

[6] A. Alb Lupaş, A note on strong differential superordinations using a generalized Sălăgean operator and Ruscheweyh operator, Stud. Univ. Babes-Bolyai Math. 57(2012), No. 2, 153-165.

[7] A. Alb Lupaş, Certain strong differential subordinations using Sălăgean and Ruscheweyh operators, Advances in Applied Mathematical Analysis, Volume 6, Number 1 (2011), 27-34.

[8] A. Alb Lupaş, A note on strong differential subordinations using Sălăgean and Ruscheweyh operators, Libertas Mathematica, tomus XXXI (2011), 15-21.

[9] A. Alb Lupaş, Certain strong differential superordinations using Sălăgean and Ruscheweyh operators, Acta Universitatis Apulensis No. 30/2012, 325-336.

[10] A. Alb Lupaş, A note on strong differential superordinations using Sălăgean and Ruscheweyh operators, Journal of Applied Functional Analysis, Vol. 7, No.'s 1-2, 2012, 54-61.

[11] L. Andrei, On some differential sandwich theorems using a generalized Sălăgean operator and Ruscheweyh operator, Journal of Computational Analysis and Applications (to appear).

[12] L. Andrei, Differential Sandwich Theorems using an extending generalized Sălăgean operator and extended Ruscheweyh operator, submitted GFTA 2014.

[13] L. Andrei, Strong differential subordination results using a generalized Sălăgean operator and Ruscheweyh operator, Journal of Computational Analysis and Applications (to appear). 
[14] L. Andrei, Some strong differential subordination results using a generalized Sălăgean operator and Ruscheweyh operator, submitted International Journal of Modern Mathematical Sciences.

[15] L. Andrei, Strong differential superordination results using a generalized Sălăgean operator and Ruscheweyh operator, submitted Journal of Computational Mathematics.

[16] L. Andrei, Some strong differential superordination results using a generalized Sălăgean operator and Ruscheweyh operator, International Journal of Research and Reviews in Applied Sciences (to appear).

[17] J.A. Antonino, S. Romaguera, Strong differential subordination to Briot-Bouquet differential equations, Journal of Differential Equations, 114 (1994), 101-105.

[18] G.I. Oros, Gh. Oros, Strong differential subordination, Turkish Journal of Mathematics, 33 (2009), 249-257.

[19] G.I. Oros, Strong differential superordination, Acta Universitatis Apulensis, 19 (2009), 101-106.

DOI: $10.7862 /$ rf.2015.3

\section{Loriana Andrei}

email: lori_andrei@yahoo.com

Department of Mathematics and Computer Science

University of Oradea

1 Universitatii street, 410087 Oradea, Romania

Received 16.03.2014 